\title{
Illumination-based Augmentation for Cuneiform Deep Neural Sign Classification
}

\author{
CHRISTOPHER REST, DENIS FISSELER, and FRANK WEICHERT, TU Dortmund, \\ Chair of Computer Graphics, Germany \\ TURNA SOMEL and GERFRID G. W. MÜLLER, Akademie der Wissenschaften und der Literatur, \\ Mainz, Germany
}

\begin{abstract}
Automated content-based search for arbitrary cuneiform signs in photographic reproductions is a challenging task in the analysis of ancient documents, a central component of which is a reliable cuneiform sign classification. We present an illumination-based approach to generate synthetic training data for cuneiform sign classification via deep neural networks to overcome common issues with the transferability of machine learning training results. Starting from an analysis of the negative impact of illumination variations in the processed cuneiform data, we employ an illumination augmentation to twodimensional (2D) training data generated from annotated 3D datasets. We demonstrate that our method is able to overcome the high visual variance of most digitized 2D cuneiform reproductions and achieve an illumination invariant generalization. The effectiveness of our approach is evaluated by its successful application to several subsets of a cuneiform script dataset with an originally poor transferability of mutual training results. Furthermore, we show that a sufficient sampling of the illumination space mostly removes the necessity to match the training data to specific target illumination conditions. The practical applicability of our approach is validated by applying it to a larger dataset, raising the overall classification accuracy by 4 percentage points to $90 \%$, resulting in a classification error reduction of $28.5 \%$ when compared to results without the proposed data augmentation.
\end{abstract}

CCS Concepts: • Computing methodologies $\rightarrow$ Object identification; Supervised learning by classification; Neural networks;

Additional Key Words and Phrases: Cuneiform, sign classification, datasets, neural networks, data augmentation, illumination, 2D-3D cross domain

ACM Reference format:

Christopher Rest, Denis Fisseler, Frank Weichert, Turna Somel, and Gerfrid G. W. Müller. 2022. Illumination-based Augmentation for Cuneiform Deep Neural Sign Classification. J. Comput. Cult. Herit. 15, 3, Article 50 (September 2022 ), 20 pages.

https://doi.org/10.1145/3495263

Christopher Rest and Denis Fisseler contributed equally to this research.

This research was supported by the German Science Foundation (DFG) within the Project "Computer-unterstützte Keilschriftanalyse (CuKa)," a cooperation between the Hethitologie Archiv, Academy of Sciences and Literature Mainz and the department of computer science at TU Dortmund university.

Authors' addresses: C. Rest, D. Fisseler, and F. Weichert, TU Dortmund, Chair of Computer Graphics, Otto-Hahn-Straße 16, Dortmund, 44221, Germany; emails: \{christopher.rest, denis.fisseler, frank.weichert\}@tu-dortmund.de; T. Somel and G. G. W. Müller, Akademie der Wissenschaften und der Literatur, Mainz, Geschwister-Scholl-Str. 2, Mainz, 55131, Germany; emails: \{turna.somel, gerfrid.mueller\}@ adwmainz.de.

Permission to make digital or hard copies of all or part of this work for personal or classroom use is granted without fee provided that copies are not made or distributed for profit or commercial advantage and that copies bear this notice and the full citation on the first page. Copyrights for components of this work owned by others than ACM must be honored. Abstracting with credit is permitted. To copy otherwise, or republish, to post on servers or to redistribute to lists, requires prior specific permission and/or a fee. Request permissions from permissions@acm.org.

(c) 2022 Association for Computing Machinery.

1556-4673/2022/09-ART50 $\$ 15.00$

https://doi.org/10.1145/3495263 


\section{INTRODUCTION}

In Ancient Near-Eastern Studies, the ongoing digitization of available cuneiform artifacts including earlier reproductions in photographic form has led to large digital collections of two-dimensional (2D) and three-dimensional (3D) cuneiform reproductions such as the Hethitologie Portal Mainz (HPM) [27] and the British Museum Collection [10]. However, unlike for the Latin script, the accessibility of these collections for content-based search methods is still an issue. So far, automatically searching large cuneiform document collections for signs or words, which is associated with the image processing tasks of localization and classification, is not possible. Enabling this would provide an immense utility value for philological work, especially with regard to the large amount of available photographic reproductions. The encountered issues can be partially attributed to the high visual variance of cuneiform signs in photographic documentations due to illumination and texture differences (cf. Figure 1(a)), the high geometric variance and complexity of cuneiform signs themselves, and the high self-similarity of individual wedge imprints. While the aforementioned factors complicate the use of traditional image processing methods for both sign localization and classification, especially with respect to generalization issues, deep learning approaches are potentially more successful in providing solutions for these challenges.

The essential building elements of cuneiform script are three-sided wedgelike imprints that were usually imprinted with a stylus into moist clay [9]. Depending on the cuneiform script variant, these so-called wedges can be categorized into a finite set of three to six types, which can be distinguished by their orientation and precise geometric shape. Multiple wedge impressions arranged relative to each other form a cuneiform sign, with the possibility of individual wedges overlapping each other as can be seen in Figure 1(a) and (b). Since cuneiform signs are the most elementary content-based information unit of cuneiform script, they are the core elements of content-based cuneiform retrieval tasks. Although the meaning of individual signs can differ depending on the cuneiform script variant and the context, being able to localize and classify cuneiform signs in digitized manuscript reproductions has a high utility value for many collation-based tasks in philology. This is particularly true with regard to an expected speed-up of manual sign retrieval.

Machine-learning methods such as deep neural networks for classification tasks usually require large amounts of training data in the form of annotated and classified samples, optimally with thousands or tens of thousands of samples per class to avoid overfitting [36]. Because many application domains do not have access to sufficiently large datasets, data augmentation techniques are applied to inflate datasets with synthetic fake data that are often generated by modifying the real data with geometric transformations (see Section 2.3). For the specific tasks of sign retrieval (sign localization and classification), training data on cuneiform reproductions can take the form of bounding boxes or polygonal shapes, as depicted in Figure 2, annotated with the respective sign names. Consequently, one core challenge in machine-learning-based cuneiform sign retrieval lies in the efficient generation of training data in form of annotated cuneiform reproductions. Since manually creating annotations of sufficient quality and quantity is a time-consuming task, suitable classification methods are required to achieve good generalization based on a comparatively small training set. This issue is exacerbated by the fact that, similarly to letters and words in alphabet-based languages, cuneiform signs consisting of fewer wedges have a tendency to be used more frequently, whereas more complex signs, occur in much smaller quantities [42]. As a result, the amount of training data available for more than half of the cuneiform character inventory is significantly reduced. This emphasizes the necessity of an effective data augmentation to achieve good generalization on cuneiform data. With the great amount of available photographic cuneiform reproductions, the potential of an augmentation method that is specially designed for cuneiform, is large.

However, due to differences of the cuneiform script compared to traditional scripts, the application of classical augmentation methods is not sufficient in this case. The main reasons for this are the three-dimensionality of cuneiform signs and the absence of contrasting lines in the writing itself. This introduces the necessity to produce contrasting lines by casting shadows into the wedge indentations to create readable photographic reproductions of cuneiform documents. Since appropriate lighting setups are created primarily with the intent to guarantee 


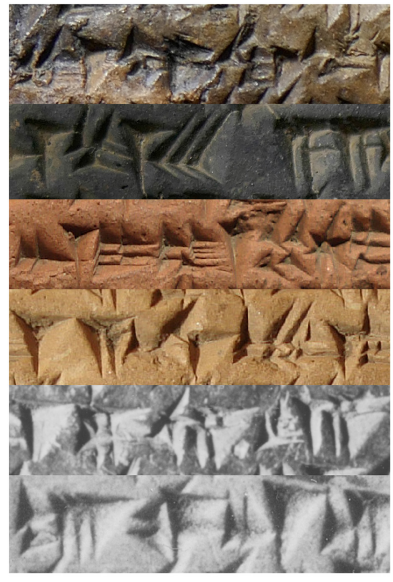

(a) High visual variance of cuneiform signs

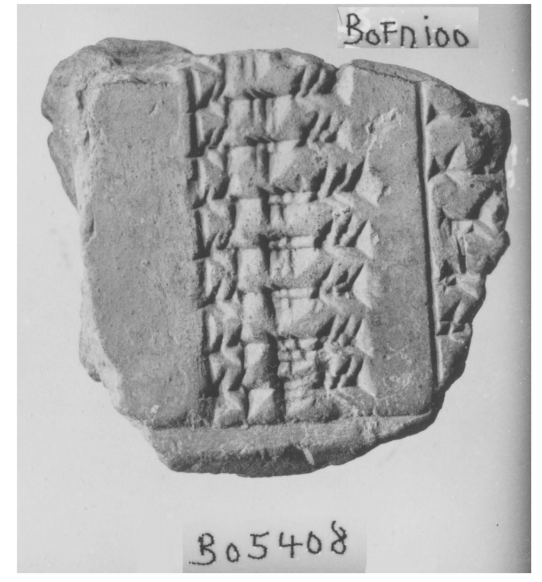

(b) Traditional black and white reproduction of Bo 5408

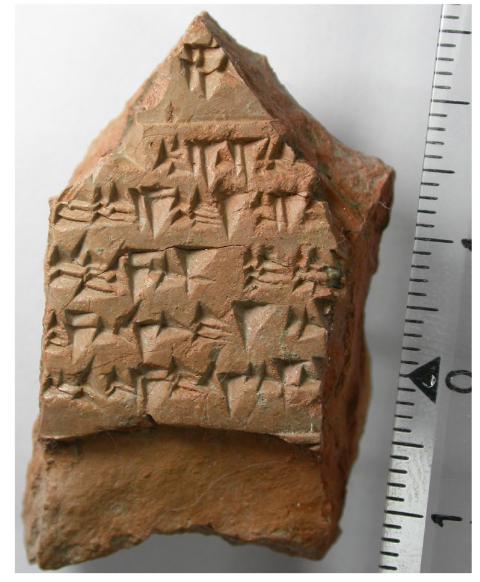

(c) Modern digital reproduction of EBo 70

Fig. 1. Exemplary traditional and modern photographic reproductions of cuneiform tablet fragments with different surface textures and illumination conditions [27].

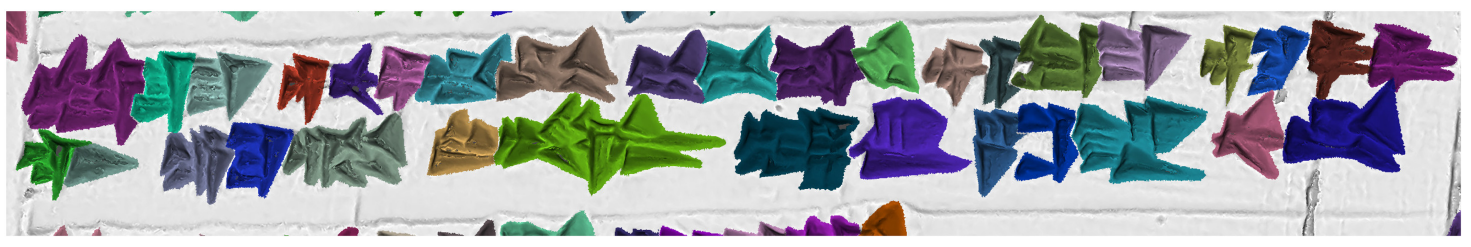

Fig. 2. Several text lines on a 3D scan of a cuneiform fragment with randomly colored, annotated cuneiform signs, where each colored contiguous polygonal area indicates one sign.

readability of the cuneiform text in the resulting images, a large variety of lighting setups exist. Consequently, the appearance of cuneiform signs can vary largely depending on the lighting setup used. Augmenting this lighting setup in the training process of machine-learning is not possible with state-of-the-art augmentation techniques, since it would require a reconstruction of the underlying geometry from the $2 \mathrm{D}$ reproductions. In contrast, it is possible to augment the lighting setup on a 3D scan of a cuneiform manuscript, which is exploited by our approach.

The main contribution of this article is therefore the introduction of a novel approach for illumination-based augmentation. This allows us to improve the transferability of deep neural cuneiform sign classification. The new approach makes it feasible to create a large amount of differently illuminated 2D representations of an annotated 3D cuneiform dataset and to incorporate this synthetic data into training datasets consisting of annotated photographic cuneiform reproductions. In this process, we also propose a post-processing technique to mitigate common issues of 3D-scanned cuneiform datasets that would otherwise negatively affect the training process. Our augmentation approach is capable of forcing an established neural network architecture to include illumination-invariant features in the learning process, thereby improving classification results on previously unknown photographic reproductions taken under different lighting conditions. We evaluate the performance (classification accuracy) based on multiple annotated cuneiform datasets with respect to classification transferability, achieved classification improvement, and suitability of the illumination augmentation. 
The article is structured as follows. In Section 2, related work is detailed that is focused on computer-aided cuneiform analysis, deep learning, and augmentation techniques. Subsequently, Section 3 describes our method. This includes our overall processing pipeline, the design of our deep neural cuneiform sign classification network, the generation of new training data using illumination augmentation, and how the augmented data are used to improve the accuracy of the classifier. A detailed description of the used datasets is given in Section 4, followed by the evaluation of the results including a comparison of the classifier with and without augmented data. Finally, in Section 5 we summarize our results.

\section{RELATED WORK}

This section briefly summarizes related work on computer-aided cuneiform analysis, deep learning, and data augmentation techniques.

\subsection{Computer-aided Cuneiform Analysis}

Digital analysis of cuneiform structures, such as wedges and signs, can be conducted based on different types of cuneiform reproductions, namely photographs, hand-drawn autographs (2D domain), and 3D representations in the form of scanned point clouds and meshes $[16,19]$. Conceptually, respective approaches can be categorized into segmentation-based methods and, like the ones presented in this article, segmentation-free methods [15, 17, 29, 32, 33]. The latter is favorable in terms of generalization, because no a priori knowledge about the content to be classified is required. Common tasks in content-based cuneiform analysis include sign classification on single-sign data excerpts, localization and classification of signs in manuscript reproductions, and query-byexample scenarios for retrieving cuneiform signs in manuscript collections. In Rothacker et al. [30], they re-factor a segmentation-free word spotting method, developed for 2D Latin script types [31], for spotting occurrences of similar wedge groups in a collection of 3D cuneiform documents. This is done by using stylized rendering to generate autograph-like 2D representations of 3D cuneiform document scans, enabling query-by-example wordspotting on 3D cuneiform documents for fast sign or word retrieval. For the extraction of cuneiform wedges from 3D-scanned manuscripts, Mara et al. [25] employ an integral invariant filter over the mesh surface and subsequently on the wedge contours to identify feature points for the internal wedge structure. Fisseler et al. [15] use a watershed approach on an indentation depth field to extract robust wedge features in form of a formalized geometrical wedge model. The extracted wedge models are then applied for statistical script analysis to identify join candidates among cuneiform tablet fragments [17] and are used by Cammarosano [8] for paleographic script classification. Unlike the above-mentioned 3D-based approaches, the availability of reliable wedge- or signlevel classification techniques on 2D photographic cuneiform reproductions is still an issue, due to their high visual variance. To circumvent these classification issues, several approaches work on hand-drawn cuneiform autographs or similar representations. This includes methods that process vectorized cuneiform, with the intermediate step of skeletonization. They use autograph-like 2D vector drawings generated from 3D-scanned cuneiform meshes [25] or raster images [26] to decompose vectorized cuneiform signs into wedge features, including the definition of sign-level similarity measures [3] or decompose spline drawings into single wedges and cuneiform signs based on part-structured models [5] and convert the vector drawings into graph representations of cuneiform signs [4]. However, neither of these methods can provide a solution for the cuneiform classification problem on photographic reproductions. Targeting photographic reproductions specifically is essential for objective philological research, since autographs represent only a subjective interpretation of a cuneiform manuscript.

\subsection{Deep Learning}

In addition to the previously mentioned mostly traditional feature-based computer vision algorithms, deep learning-related methods are becoming increasingly important. They offer the possibility to solve much more 
complex problems, usually at the cost of requiring much more computational resources [24]. Deep learning [18] is based on the paradigm of neural networks, a data structure made from layers of interconnected artificial neurons, which propagate information from an input layer through one or more hidden layers to an output layer. The information propagation inside the network is controlled by weights in the neurons, which have to be determined in a training phase to produce the desired output function. Deep neural networks can be trained to approximate any mathematical function. In case of classification, neural networks learn an unknown function that approximates the true mapping of the input to the output classes. During the learning process, the parameters are optimized to obtain the best approximation of the true mapping. Although the design of a neural network for classification purposes is problem specific, most networks can be used on a broad range of similar problems, making existing image classification networks a good baseline for cuneiform classification. Popular respective network designs include ResNet, DenseNet, VGG, AlexNet, and FixEfficientNet [21, 23, 35, 37]. While deep neural network architectures are applied extensively in various fields of interest, only few deep learning-related methods have been applied to cuneiform data so far. One example on scanned 2D cuneiform autographs is the automated generation of cuneiform transliterations based on various basic machine learning methods to classify sequences using context-sensitive and context-insensitive variants by Bogacz et al. [6]. Kriege et al. use wedge models from Fisseler et al. [15] to construct a 3D graph structure for cuneiform signs [22]. For sign classification, the performance of the SplineCNN by Fey et al. [14], a graph convolutional network, is compared against the graph edit distance in combination with a nearest neighbor classifier. Bogacz et al. combine PointNet++ and SplineCNN to predict the time period of cuneiform tablets represented by 3D meshs [7]. In Rusakov et al. [32], they evaluate the use of cycle-consistent adversarial networks to transfer projections of 3D cuneiform tablet fragments to autograph-like images for an artificial inflation of existing annotated cuneiform training datasets. While this represents a promising approach for cuneiform sign classification on 3D datasets and autographs, it again provides no general solution for the cuneiform classification problem on photographic reproductions.

\subsection{Data Augmentation}

The availability of comprehensive annotated data is a basic prerequisite to reliably train supervised deep learning methods. However, in many fields of interest, such as cuneiform analysis, the amount of available data is comparatively small and the generation of new annotated data is work intensive [34]. Therefore, regularization techniques [28] are often used when training neural networks to overcome the small size of the training dataset. One of these techniques is data augmentation, which inflates the dataset size using affine transformations [20]. Frequently used image augmentation techniques include mirroring, rotation, random cropping, adding random noise, and blackening, to name a few. Due to the characteristics of handwriting, commonly used augmentation techniques in digital handwriting analysis with neural networks are sheering, color variation, and rotation $[1,2,38]$. For localization tasks, traditional augmentation techniques also include flipping, random erasing and translation $[12,41]$. In contrast to classification, the annotations for localization tasks additionally contain positional information, defined, for example, as a bounding box or a mask. Depending on the augmentation used, this positional information has to be adjusted as well. Approaches are based, for example, on removing parts of the training image to force the neural network to focus on more descriptive features in the data instead of focusing on a few overly present features [41]. In contrast, translation augmentation reduces the likelihood that objects are centered in the training image and therefore avoids positional bias [34]. However, while data augmentation has been commonly used in many fields of application, to the authors' knowledge no augmentation method is currently available that takes into account the specific characteristics of cuneiform data. The differences of cuneiform writing as opposed to traditional scripts arise from the three-dimensionality of cuneiform signs and the absence of contrasting lines in the writing itself. Augmenting the complex illumination and shading properties of three-dimensional cuneiform signs on photographic reproductions is not possible with the previously described augmentation techniques, since it would require a simulation of the lighting setup and a 


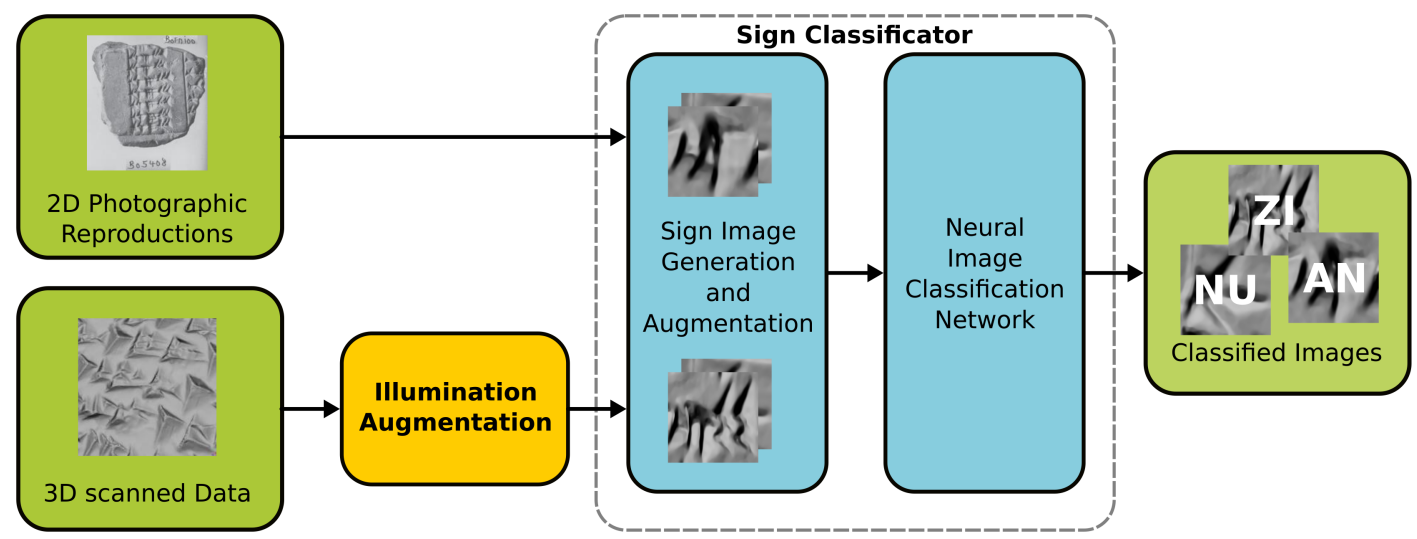

Fig. 3. Processing pipeline of our sign classification architecture for $2 \mathrm{D}$ photographic reproductions and 3D-scanned cuneiform manuscripts. The core components of our method are the Illumination Augmentation (Section 3.1) and the Sign Classificator (Section 3.2).

reconstruction of the underlying geometry from the 2D reproductions. This issue is avoided by our method, by incorporating 3D-scanned cuneiform data. A state-of-the-art method by Deschaintre et al. is, to some extend, comparable to our method [11]. Using rendered images with varying textures, that are lit using different lighting scenarios, as done in this work, the authors train a neural network to capture Spatially-varying bi-directional reflectance distribution functions (SVBRDFs) from single images. It is therefore possible to capture texture details from images using the trained neural networks. Even though the method is not designed for data augmentation, these SVBRDFs could be used to augment existing data using varying lighting scenarios while the textures are captured from cuneiform images. Compared to our method, the usage of neural networks might erase potentially available features when capturing the SVBRDFs. It is unknown to the authers how well this technique would work on cuneiform data.

\section{METHOD}

The goal of our method is to achieve a reliable classification of cuneiform sign images taken from photographic reproductions, despite the presence of the previously mentioned illumination-associated issues, by employing a problem-specific illumination augmentation. Consequently, our cuneiform sign classification architecture consists of a sign classificator containing a neural image classification network with respective input image preprocessing and a generator for synthetic illumination augmented training images.

One core challenge in this design lies in the efficient generation of illumination augmented cuneiform sign training images from 3D-scanned cuneiform datasets. The training images not only have to be illuminated in a way that allows to sufficiently sample the expected input illumination parameter space and therefore achieves good generalization but also to match the visual style of the targeted photographic reproductions.

Additional equally important challenges are the consistent pre-processing of both the photographic cuneiform sign images and the synthetic training images, to determine an appropriate complexity of the employed image classification network and to equip the classification network with a sufficient amount of traditional image augmentation. Figure 3 shows our complete processing pipeline for addressing these challenges, whose components will be detailed in the following.

\subsection{Illumination Augmentation}

Common augmentation techniques for script classification training data include translating, scaling, and rotating the respective script images. While image translation and scaling can be naturally applied to cuneiform data, 
rotational augmentation exhibits an important difference as opposed to script data written on flat media like paper. This results from the fact that in most photographic cuneiform reproductions, the script features are emphasized by shadow casting from a directional light source. As can be seen on the photographs in the HPM the light source is usually positioned at an angle, so that the rims of the wedges cast hard shadows into the indented inner area of the wedges. This is especially true for reproductions from the pre-digital age like the large group of reproductions from the HPM. As a result, otherwise hard to see geometric wedge features become visible in the photographic reproductions. Consequently, when the script changes its line direction on a cuneiform document, the global illumination direction remains unchanged. Thus, just rotating an image of a cuneiform sign will not match the situation resulting from a locally rotated writing direction on an actual cuneiform document. Conversely, rotating an image of a cuneiform sign will inadvertently lead to an implicit rotation of the light direction. This means that the illumination must be taken into account when applying rotational augmentation. But in addition to its linkage with the script rotation, illumination is also an important augmentation parameter. While flat cuneiform manuscripts can be expected to exhibit an uniform illumination regardless of the location, it can vary significantly on curved writing surfaces. Moreover, the illumination direction changes between individual photographic reproductions, since it is usually adjusted manually to optimize the resulting visual detail enhancement on the individual artifacts. A comparatively constant illumination can thus only be expected between photos taken in consecutive order using the same camera and lighting setup. But even then, the illumination could vary because of different orientations of the tablet fragments with respect to the camera or the light source. These differences become even more pronounced when viewing photographic reproductions from different sources. An additional factor that could be augmented on cuneiform data is connected to the surface texture of the tablet fragments. Fragment surfaces can be rough or smooth and exhibit a variety of color variations such as clay, color, burn marks, chemical corrosion, and impurities of the clay. In contrast to the illumination, these factors on average have a much smaller influence on the recognition-relevant appearance of the wedges, which is mainly based on visible edges. Likewise, negative influences of the employed photographic system like image noise or lens distortion can usually be neglected. This can be attributed to the fact that cuneiform photographs are usually taken in a static situation, which allows the use of low ISO films and of high quality, low-distortion lenses. We will therefore concentrate our efforts on the augmentation of illumination.

One way to augment the illumination on cuneiform reproductions is to take photos of cuneiform fragments under varying lighting conditions. While this is possible with automated setups like the Leuven-Light-Dome [39], it is a time-consuming process, requiring extended amounts of time with the actual cuneiform fragment. In addition to that, the exact type of illumination augmentation must be planned in advance and cannot be changed after the data acquisition. These issues can be resolved by using existing 3D scans of cuneiform manuscripts as a base for generating the illumination augmented training samples. Rendering the 3D scans while illuminated by a virtual light source that is located at different positions above and around the fragment creates images with augmented illumination from parameterizable elevation and azimuth angles. This way, different illumination augmentation techniques can be evaluated without having to capture the original cuneiform manuscript again.

An efficient illumination augmentation should mimic the illumination variations that can be found in common photographic cuneiform reproductions as closely as possible. At the same time, keeping the sampling density of the parameter space low could help with minimizing the additional training efforts. We chose a subset of the black and white photographic collection of the HPM [27] as the design template for our synthetic dataset, since it is representative for a large portion of the available photographic cuneiform reproductions for Hittite cuneiform. Aside from being monochrome, these reproductions are characterized by a similar type of acquisition setup, where the camera points perpendicular onto the writing surface and the writing direction is aligned from left to right being approximately horizontal. Possible light directions in this setup can be defined in a hemispherical coordinate system by the parameters of azimuth and elevation. As shown in Figure 4, the azimuth $\alpha$ in this setup describes an angle lying in the plane defined by the writing surface, while the elevation angle $\theta$ denotes the angle between the light direction $d$ and this plane. For generating images of cuneiform signs, this hemispherical 


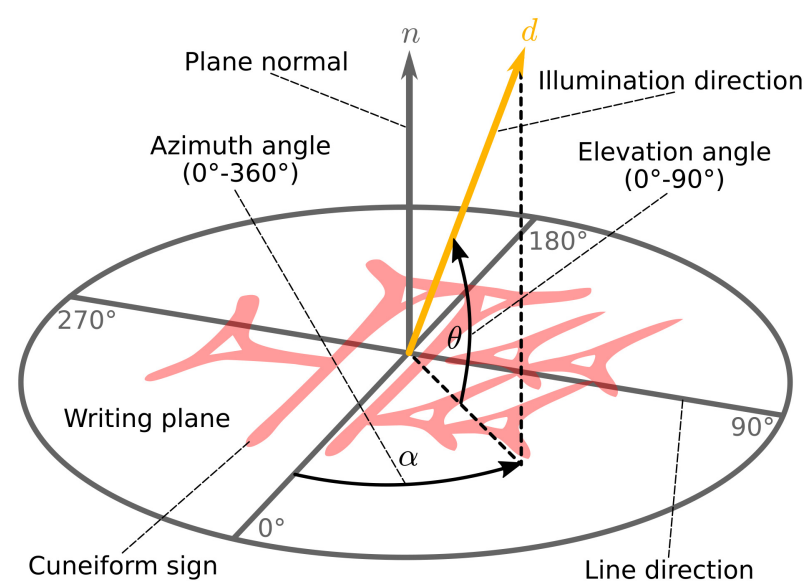

Fig. 4. The illumination direction, defined via azimuth and elevation angle with respect to the line direction.

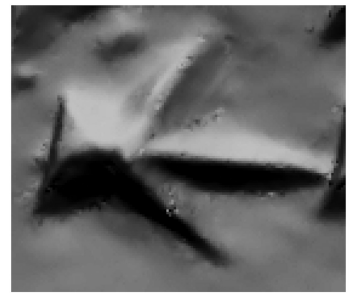

(a) Azimuth angle $0^{\circ}$

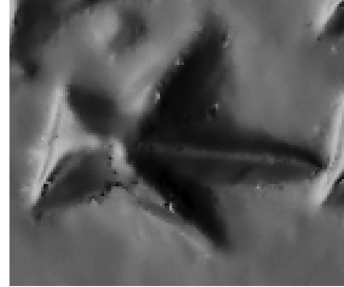

(b) Azimuth angle $90^{\circ}$

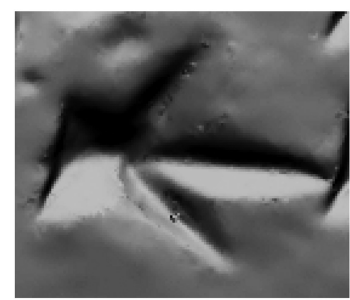

(c) Azimuth angle $180^{\circ}$

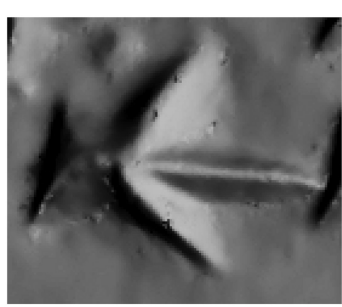

(d) Azimuth angle $270^{\circ}$

Fig. 5. Four rendered illumination variations of the cuneiform sign $N U$ that result in a different visual appearance. Most geometric edges are not visible in all images and many wedge faces completely change between dark, medium and high brightness between the images.

coordinates system can be defined by using the normal vector $n$ of a local approximation of the writing surface as the plane normal and the zero value of the azimuth angle being tied to the local writing line direction.

The choice of illumination directions is based on the observation that too large elevation angles generate no shadows, while elevation angles close to $0^{\circ}$ cast shadows filling the entire wedges. In accordance with that, the illumination directions in real photographic reproductions can be observed to exhibit elevation angles close to $45^{\circ}$. Consequently, we choose an elevation angle of $45^{\circ}$ and vary the azimuth angle from $0^{\circ}$ to $360^{\circ}$ in $N$ steps to obtain the illumination augmented images for each sign. In addition to that, the shading is parameterized to match the appearance of the black and white photographic reproductions. A resulting example for four illumination directions of the cuneiform sign $N U$ can be seen in Figure 5. The generated images are then post-processed to compensate for scan inherent issues, as will be detailed later in Section 4, and subsequently passed to the sign classificator.

\subsection{Sign Classificator}

Since the design and adaptation of a neural network to specific classification tasks and the assessment of its performance and suitability are complex issues of its own, we adopt an existing and sufficiently well tested neural image classification network and embed it in our illumination augmented cuneiform sign classification architecture. We opted to use ResNeXt [40], a well-tested image classification network design, which provides 


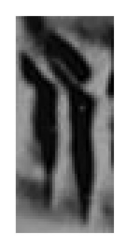

(a) Sign A

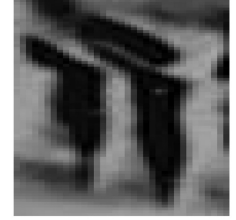

(b) Augmented A

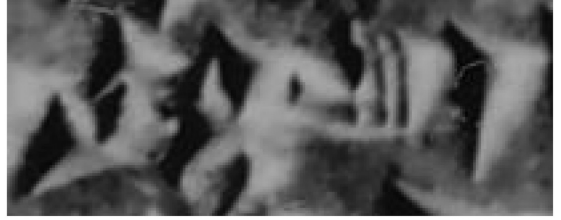

(c) Sign EREN

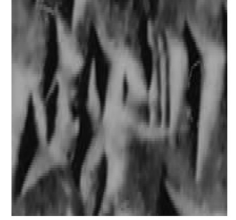

(d) Augmented EREN

Fig. 6. Different aspect ratios in photographic cuneiform signs and it's effects on augmentation.

high flexibility and adaptable complexity levels. Using a network complexity that matches the complexity of the classification problem to be solved is a necessity to avoid under- or over-fitting during training. Therefore, ResNeXt is available in different network depths, allowing an adaption to the desired degree of complexity. For our application, we specifically use ResNeXt-18 and ResNeXt-50, where the number denotes the network depth, which both require input images of size $224 \times 224$ pixels. Since our input data consists of grayscale images and contains only cuneiform-specific content, the ResNeXts are trained from scratch on cuneiform data only. As for the required learning parameters of the neural network, during our training, a batch size of 8 and a constant learning rate of 0.001 is used.

As a prerequisite to training the network with photographic or rendered images, several preprocessing steps have to be performed. Initially, the cuneiform signs in the 3D meshes are rendered to 2D images while augmenting the illumination as described in Section 3.1. The photographs and rendered images are then cropped to rectangular bounding boxes that contain single signs. This ensures that as little of other cuneiform signs as possible is present in each image section, as this would make the training process more difficult or, in the worst case, change the label of the cuneiform sign. In contrast to the photographs, the rendered data are additionally post-processed to reduce rendering artifacts as will be detailed later on in Section 4 . The images are then converted to grayscale and their dimensions are scaled to the uniform square size of $224 \times 224$, required by the ResNeXt. As shown in Figure 6, this can cause severe distortions of the cuneiform signs due to different aspect ratios of the sign classes. However, distortion at this point is preferable over a simple extension of the image section to bounding squares. This is due to the former retaining most gradients in the image and the latter often resulting in interfering wedge structures of surrounding signs being included in the cropped images.

Regardless of other augmentations, random cropping is applied to the image data to achieve a slight positional invariance during training. Random cropping should also help the network architecture to handle slightly reduced data, similar to the use of dropout. The reason for using random cropping instead of drop-out layers is that it allows some control over which data can be omitted. As opposed to positional augmentation, no rotation augmentation is used here, because the images of the cuneiform signs are aligned almost horizontally in our training data. Thus, rotation augmentation would introduce an unnecessary degree of freedom. Before the image data are fed into the neural network, a normalization of the color values is performed.

Our subsequent training process consists of two main steps. To generate a database to compare further experiments with, the networks are first pre-trained on photographic cuneiform data until convergence is achieved. In the second step, the influence of rendered data on the performance of the net is evaluated. For this purpose, the training data are supplemented by illumination augmented rendered data and the nets are trained again until no further gains in accuracy can be observed. The rendered data are supplied in groups that contain different illumination directions to evaluate the influence of different lighting setups on the quality of the classifier.

\section{RESULTS AND DISCUSSION}

To evaluate our hypothesis that illumination-based augmentation can improve the accuracy of a deep neural classifier on cuneiform images, the neural network is trained several times with different sets of data. In this 
process, the training data are supplemented by artificially rendered images of additional illumination directions to evaluate whether this leads to an increased classification accuracy. We will start with a detailed description of our dataset in Section 4.1. In Section 4.2 we establish a baseline and show that the choice of our subset is reasonable. Section 4.3 will then present and discuss the classification improvements achieved by our illumination augmentation.

\subsection{Experiments and Dataset}

The design of our dataset ${ }^{1}$ is motivated by the objective to show that the performance of a neural network can be improved by adding illumination augmented rendered data. To model a real-world application scenario, we have chosen to use a dataset, representative for Hittite cuneiform from the Hethitologie Archiv (accessible via the HPM [27]). Those representative aspects include the direction of illumination, picture recording conditions, and character distribution as detailed in Section 3. Our dataset consists of 13 cuneiform tablet fragments represented by 63 fully annotated images and one annotated 3D scan taken from one of the fragments in 2008. The Hittite cuneiform script can subjectively be described as "tidy" in general, since it was written by professionally trained scribes. In this sense, it is different from Old- and Neo-Babylonian business documents written in cursive script, which were not always written by professional scribes. In spite of this, the Hittite cuneiform (as with all other variants of Sumero-Akkadian cuneiform script) is heterogeneous as to the variants of graphemes used, the angles of wedges, and the line (and sign) spacing due to factors such as scribal preferences, difference in text genres, and scribal trends in different epochs. Since Hittite cuneiform signs belonging to the same word are usually written close together (so as to contact one another), the right and left edges of a sign may look different depending on the neighbouring sign (or the lack thereof). To obtain results that may be applicable to the Hittite text corpus in general, a group of texts signed by different scribes and palaeographically attributed to different epochs within the Hittite palaeography were used for this study. However, the selection of texts does reflect a certain bias toward festival and ritual texts. This bias stems, on the one hand, from the general bias within the Hittite corpus toward these genres, and, on the other hand, from the fact that the texts belonging to these genres were more often signed by the scribes, which allowed us to test the effectiveness of this method across at least nine different scribal hands. The used tablets are written in a Hittite cuneiform variant with non-cursive writing, a uniform surface texture and a medium to low sign overlap. The overall structural damage to the tablets in the dataset is low, although a moderate number of cracks and wedge contaminations by fill material exist. With respect to the image quality, the dataset includes neither remarkably bad nor remarkably good photographs. Thus, the amount of image noise, blurriness and over-/underexposures is moderate. The 3D scan in our dataset contains no large geometric scanning errors, but a fair number of geometric issues, like small holes and other scanning induced errors at wedge scale.

An image of a fragment can contain a large number of signs and each single annotation consists of a polygonal outline of a sign with an associated class attribute. This outline is converted into a writing direction aligned rectangular bounding box, resulting in an image section of a sign representing its character class. For the 2D data, the writing direction alignment is obtained by exploiting the knowledge that the photographic reproductions were taken with the writing direction being aligned horizontally. On the 3D scan, the writing direction is specified manually in terms of a spline-derived interpolated vector field so that the rendering process creates images that contain horizontally aligned cuneiform signs.

Our annotated 2D dataset contains a total of 22.800 annotated cuneiform signs, comprising 228 of approximately 375 sign classes of the Hittite corpus. Including sign variants of these 375 sign classes, the Hittite corpus contains around 3.500 sign classes. For our experiments, the 1.678 annotated 3D signs in our dataset are artificially illuminated. As already detailed in Section 3, the lighting elevation angle $\theta$ is fixed at $45^{\circ}$, leaving the azimuth as the degree of freedom. With an equidistant sampling of the azimuth angle $\alpha$ by 13 directions, a total

\footnotetext{
${ }^{1}$ The dataset is available at: https://graphics-data.cs.tu-dortmund.de/downloads/cuneiform/Cuneiform_Dataset_JOCCH.zip.
} 
Table 1. Overview of the Photographic Dataset with Respect to the Contained Fragments, the Associated Images, Signs, and Approximate Lighting Direction Denoted by the Azimuth Angle

\begin{tabular}{|c|c|c|c|c|c|c|c|c|c|c|c|c|c|}
\hline Fragment & $\underset{\mathbb{1}}{\stackrel{1}{1}}$ & 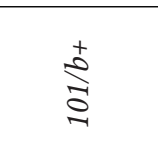 & \begin{tabular}{l}
+ \\
\multirow{+}{+}{} \\
0 \\
0
\end{tabular} & $\frac{+}{N}$ & $\frac{+}{\stackrel{+}{\sigma}}$ & $\stackrel{-}{m}$ & $\stackrel{\varpi}{\triangleq}$ & $\underset{\substack{+\infty \\
\infty \\
+\infty}}{+}$ & 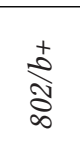 & $\begin{array}{l}\infty \\
\infty \\
\infty\end{array}$ & 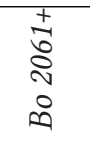 & $\begin{array}{l}\text { ָे } \\
\text { N } \\
0 \\
0\end{array}$ & 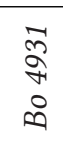 \\
\hline \#Images & 2 & 14 & 6 & 4 & 3 & 2 & 2 & 11 & 7 & 2 & 2 & 4 & 4 \\
\hline \#Signs & 2877 & 2926 & 3091 & 2633 & 741 & 596 & 429 & 2752 & 1824 & 327 & 2463 & 1329 & 805 \\
\hline Azimuth $\left[^{\circ}\right]$ & 270 & $180 \& 225$ & 225 & 270 & 270 & 270 & 270 & 270 & 270 & 250 & 250 & 250 & 250 \\
\hline
\end{tabular}

amount of 21.814 illumination augmented 2D images is obtained. Regarding the distribution of cuneiform sign classes, our whole dataset is highly unbalanced (for a list of classes and their distribution see Figure 14). This is expected, since in Hittite cuneiform some signs are far more common than others, similar to syllables in Latin script. A common approach in deep learning for dealing with unbalanced datasets is the oversampling (or undersampling, respectively) to adjust the class distribution. However, these techniques cannot be employed here, because in some cases there is just a single image available per class, so the neural network would not be able to generalize well on the basis of a single image. Instead, we opted to restrict the number of characters used, as will be detailed later on. The cuneiform fragments for the dataset are selected specifically to allow to clearly attribute any observed classification improvements to the incorporation of illumination augmented data. Further selection criteria are a sufficient size of the fragments, the availability of a sufficient amount of signs in 2D and 3D, and the presence of several differing directions of light in the photographs. An overview of the dataset can be seen in Table 1. Most of the photographs contain shadows induced by a light source located to the left of the tablet, corresponding to an azimuth angle of approximately $250^{\circ}$ to $270^{\circ}$. In some cases, the light source is located at the top or upper left of the tablet, corresponding to azimuth angles $\alpha$ of $180^{\circ}$ to $225^{\circ}$, which results in differently cast shadows. This small subgroup of data consists of 2 fragments and is used for selective cross-validation of single-fragment training results and for validation of the illumination augmented data.

The tablets in the dataset used for initial transferability evaluation of the learning results are $710 / b, 101 / b+$, and Bo 49+, which all exhibit different lighting angles but contain a similar number of signs. The tablet $710 / b$ is a single fragment including an obverse and a reverse side and is, consequently, represented by 2 photographs. It is chosen to ensure transferability between the two domains, since it is the only tablet annotated in both $2 \mathrm{D}$ and 3D. On both photographs, the light source is located to the left of the tablet. Tablet $101 / b+$ contains 14 fragments where the light source is located at an azimuth angle in the range of $180^{\circ}$ to $225^{\circ}$ and tablet $B o 49+$ has several fragments where the light source is located at an azimuth angle of $225^{\circ}$. Since we want any improvements in the performance to be due to the addition of the rendered data, we need to ensure that properties other than the direction of light affect the results as little as possible. This is achieved among other things by choosing fragments from the same group of Hittite manuscripts and a comparatively similar type of hand writing.

\subsection{Baseline Analysis}

To determine the influence of illumination augmented data on the classifier accuracy, we need a baseline trained exclusively on real photographs. For this purpose, we have used images of three tablets with different lighting scenarios as mentioned in Section 4.1. The transfer quality without illumination augmentation between the selected tablets is evaluated by performing cross-validation, where for each tablet a ResNeXt classifier is trained on the images of its fragments and the images from the remaining two tablets were then used as test sets.

Results of this cross-validation can be seen in Figure 7, where the performances are shown for each training iteration. The expected poor transfer quality between the learned features from different tablets is indicated by 


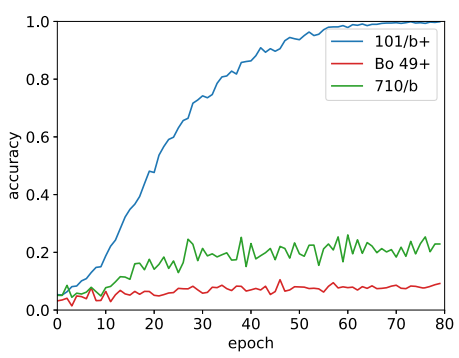

(a) Trained on tablet $101 / b+$

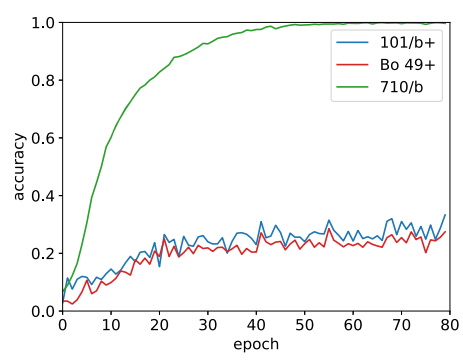

(b) Trained on tablet $710 / b$

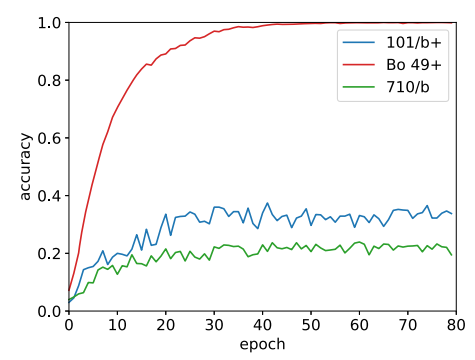

(c) Trained on tablet Bo 49+

Fig. 7. Cross-validation on tablets $710 / \mathrm{b}, 101 / \mathrm{b}+$, and $\mathrm{Bo} 49+$. The fast rising curve represents the accuracy on the training set in each graph.

the large gap between the training curves and the test curves in the graphs after convergence of the training curves is achieved. Possible reasons for the poor transferability can be the lighting setup, image artifacts, different sign geometry, and a different sign distribution among tablets. In the following, we will discuss these options to minimize non-illumination-related influences.

Since all tablets are from the same manuscript group of Hittite documents, the geometry of the signs can be expected to be similar enough to have no significant influence on the performance.

To examine the influence of the cuneiform sign distribution on the accuracy, we determined the frequencies of the sign classes on the tablets that were chosen for our evaluation scenario. This distribution, as can be seen in Figure 14, is unbalanced and some classes do not occur on all tablets. To ensure a fair evaluation, character classes that just occur in one of the two test sets, but not in the training set, were removed from the test sets, because the neural network could not be trained for these classes.

As a further step to equalize the data, only those character classes were used that occur with sufficient frequency. In this case, we decided to evaluate a 90:10 and a 80:20 heuristic to remove character classes that occur too rarely. As a result of the heuristic, character classes with, e.g., less than 10 occurrences are not included in the test data. Results of the cross-validation after trimming the datasets can be seen in Figure 8 for the example of training on tablet 710/b. As can be seen in Figure 8(a), just removing mutually non-existing classes already represents a considerable improvement over Figure 7(a). Not taking into account the least attested $10 \%$ of sign classes provides an additional improvement in accuracy, while increasing this proportion to $20 \%$ yields no advantage.

To further inspect possible reasons for the low transferability, we measure the influence of different amounts of photographic image artifacts such as noise on the accuracy. For this, the amount of noise in our photographic data is equalized by applying a low-pass filter. The resulting performance gain of only 3 percentage points is significantly lower than the one achieved by taking the sign distribution into account, suggesting that the influence of image noise is negligible.

Consequently, as the remaining factor, we expect the illumination to exert a significant influence on the transfer accuracy of the neural network. Initial evaluations of the suitability of the generated data showed an accuracy of only 0.05 when training with rendered 3D data of tablet $710 / \mathrm{b}$ and testing on photographic data of the same tablet. This low accuracy is, however, not a result of a general data incompatibility, but the result of data quality issues. This is due to the fact that 3D-scanned meshes from cuneiform artifacts, that were scanned with older 3D scanners, often contain geometric errors that are introduced during the scanning process. Scans, that are taken with todays state of the art scanners, show less of these defects; however, a lot of lower quality 3D scans exist in digital cuneiform collections. These errors are data acquisition issues that create artifacts like holes and non-2-manifold or overlapping geometry that are not present in real data. Consequently, rendering this faulty geometry creates strong edges in the cuneiform wedges that are non-existent on the real artifact and the photographic reproductions. This leads to an ill-conditioned training process, because neural networks heavily focus 


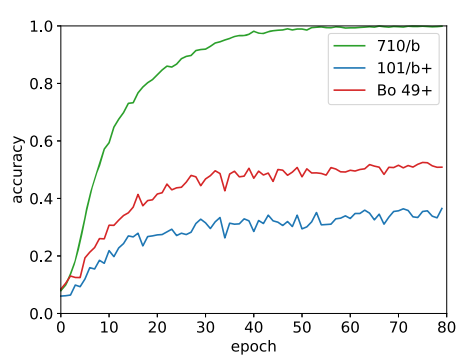

(a) Trained on the top $100 \%$ of $710 / \mathrm{b}$

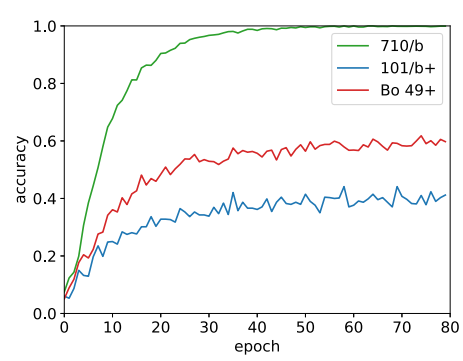

(b) Trained on the top $90 \%$ of $710 / \mathrm{b}$

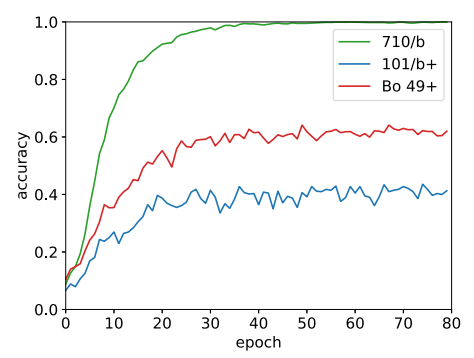

(c) Trained on the top $80 \%$ of $710 / \mathrm{b}$

Fig. 8. Effects of dataset equalization via removal of mutually non-existent classes for training on tablet $710 / b$ and additionally not considering different amounts of seldom attested sign classes.

on gradients. As a result, it is mandatory to repair the rendered data to reduce these artifacts. However, employing mesh repair on the 3D model is not easily possible without introducing further defects and data biases. Instead, we chose to repair the data using a 2D image post-processing step composed of applying mean curvature blur [13] and tone mapping to make the resulting images look more like real photographs. Figure 9 shows the starting point and the result of this data post-processing where the post-processing clearly mitigates the numerous geometric artifacts inside the wedges. Reevaluating the transfer accuracy between photographic and the repaired rendered data now shows an up to 10 -fold better accuracy.

We additionally evaluate if the rendered lighting directions work as expected. For this, the network is trained on photographs of the front of tablet $710 / b$ and tested on rendered data of the same tablet, as can be seen in Figure 10. While the accuracy of the classifier remains low when images with mismatching light conditions are used (green line), the accuracy exceeds 50\% when the conditions match (blue line). Because the rendered data still differ from the photographs, we did not expect high accuracy. Since the rendered data are only supposed to contribute information regarding the illumination direction to the real data, the rather low accuracy does not pose a problem. Using the post-processing method, it is therefore possible to evaluate the influence of differently illuminated tablets on the accuracy of a neural classifier, which will be done in Section 4.3.

\subsection{Evaluation of the Augmentation Method}

Based on the experiments in Section 4.2, we can ensure that improvements on the accuracy of the neural classifier are predominantly due to the different illuminations of the newly added rendered training data by our novel augmentation method.

For the training of our classifier, we generated rendered data from 13 different illumination directions $\mathcal{L}=$ $\left\{l_{1}, \ldots, l_{13}\right\}$ and partitioned them into a set of groups $\mathcal{G}=\left\{g_{1}, \ldots, g_{6}\right\}$, where each group $g_{j}$ consists of the synthetic data of several adjacent illumination directions $l_{i}$ as shown in Figure 11. The groups were defined in such a way as to allow meaningful evaluation scenarios with respect to the existing real imagery in our dataset.

To evaluate the effects of our illumination augmentation we chose three tablets with non-matching lighting directions as our starting point. Tablet $710 / b$ is used as the training dataset, since it is the only tablet with $2 \mathrm{D}$ and 3D annotated data. The photographs of this tablet contain illumination directions corresponding approximately to the light directions $l_{10}$ and $l_{11}$ from Figure 11. As test datasets, we chose tablets $101 / b+$ and Bo $49+$, which exhibit a greater illumination variance and are lit from directions in the range of $l_{7}$ to $l_{10}$. This motivates the light direction grouping strategy with $g_{1}$ to $g_{6}$. The groups $g_{1}, g_{2}$, and $g_{4}$, with light directions that do not correspond to those of the test data, and $g_{3}, g_{5}$, and $g_{6}$, whose directions of light correspond to those of the test data. Specifically the group $g_{6}$ is a close approximation of the lighting directions in the test tablet Bo 49+, while the photographs of 


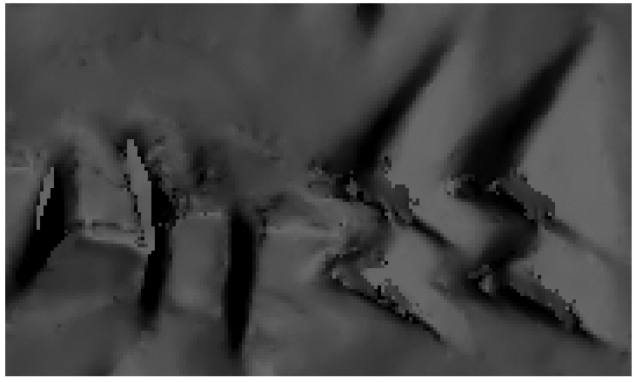

(a) Rendered image without post-processing showing many mesh-error-induced artifacts.

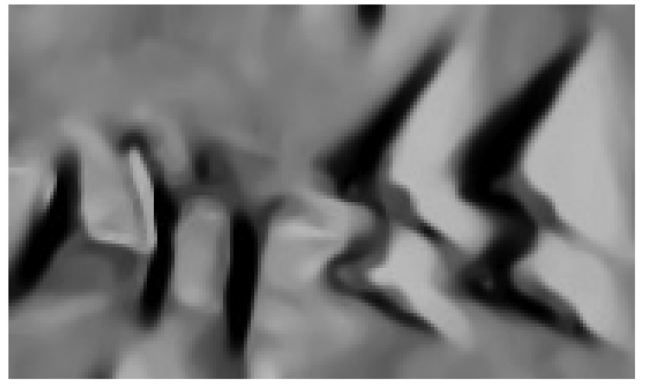

(b) Post-processed rendered image with mean curvature blur and tone mapping applied.

Fig. 9. Effect of the post-processing on a rendered image. Mean curvature blur must be applied with care, since the image artifacts should be removed while at the same time the effect on the gradients should be as small as possible.

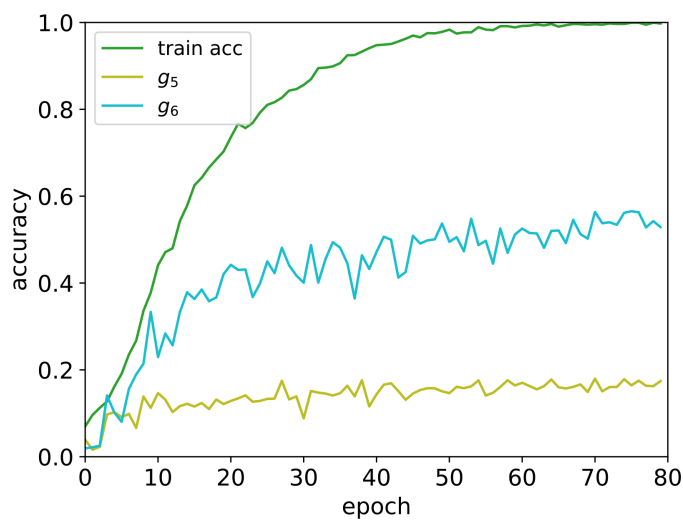

Fig. 10. Effect of the position of the light source on the performance with a non-matching light direction (olive colored line, $g_{5}$ ) and a matching light direction (light blue line, $g_{6}$ ).

test tablet $101 / b+$ contain directions from $g_{5}$ and $g_{6}$. This grouping allows testing the augmentation with closely matching, partially matching, mildly off-valued and completely off-valued light directions.

Consequently, no performance gain is expected when the groups $g_{1}, g_{2}$, and $g_{4}$ are added to the training data of the classifier, since the non-matching light directions are not expected to contribute any useful information to the training dataset with regard to the illumination of the test data. The groups $g_{3}, g_{5}$, and $g_{6}$, however, contain lighting information similar to that in the photographic test data, which does not occur in the photographic training data. So a performance gain of the neural network is expected when incorporating synthetic data from these groups.

For the actual experiments, multiple ResNexts were trained with different training sets, where each training dataset consists of the photographic data of tablet $710 / b$ and the rendered data from one group $g_{j}$. Two test sets were used, one containing all images of tablet $101 / \mathrm{b}+$, the other one containing all images of tablet Bo $49+$. Figure 12 shows the observed influence of the groups $g_{j}$ on the performance of the ResNext. In both graphs, the bold gray-colored line represents the baseline from Figure 7(b).

For both test datasets, no significant increase in quality with additional data from $g_{1}$ and $g_{2}$ can be observed. On the contrary, the accuracy tends to be even lower than the baseline for these training datasets. This results from the fact that these groups provide no useful illumination information for the classifier with respect to the 


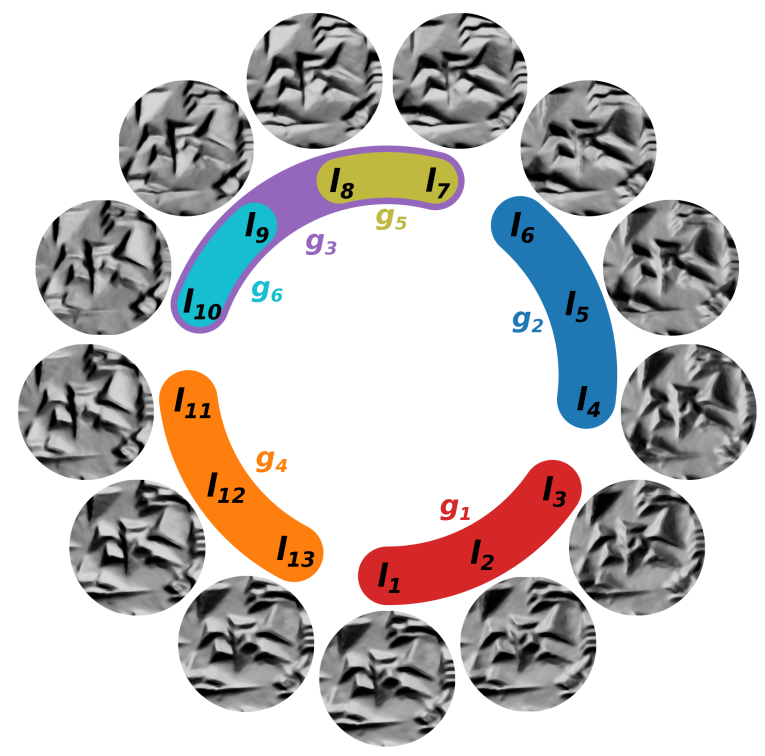

Fig. 11. Visualization of the 13 directions of the virtual light sources used during rendering. Colored sections $g_{j}$ represent lighting groups that include synthetic images of the associated light directions.

test sets as these were illuminated from different angles. As a consequence, the generalization of the network suffers.

However, for the groups $g_{3}$ and $g_{5}$ with the best matching light directions, a positive change in the accuracy is achieved. This significant improvement is based on the light directions in the test data not being present in the non-augmented training data $\left(l_{10}\right.$ and $\left.l_{11}\right)$.

Group $g_{6}$ also contains comparatively good matching light directions that, however, do not lead to a big improvement in accuracy. This could be due to the augmented data being too close to the non-augmented baseline data to force a generalization toward the contained light directions.

The overall better performances for the tablet $B o 49+$ can be attributed to the non-augmented training data being a better match for the test data than for the tablet $101 / b+$. This, in addition to the sharper images of $B o 49+$ with visible surface texture is also a plausible explanation for the more noisy performances on Bo $49+$. As can be seen in Figure 12(b), the noise is most noticeable for the illumination directions contained in the groups $g_{4}$ and $g_{5}$, which are neither a very good nor a very bad match to the test data.

Finally, when incorporating all illumination directions, it can be seen that the chosen sampling distance of the azimuth angle is sufficient to force a good generalization. The resulting performance exceeds all previous results, even the ones from augmentation with the best matching lighting directions. It can be concluded that the network learned to become invariant to the light direction and 3D generated training data can be used to improve the quality of neural networks trained on photographic image data.

So far, we have only used the selected subsets of our data for validation. To examine the potential effects of illumination augmentation on a larger dataset with a greater variance of contained illumination directions, we extended the training data to our whole dataset. For this, a ResNeXt is pre-trained on $80 \%$ of the entire dataset, converging to a base accuracy of about $86 \%$. Groups of rendered data were then added to the training set and the network was trained for another 50 epochs. Figure 13 shows the achieved improvements. The blue and red curves represent light directions that do not exist in the photographic images of the training data and the results 


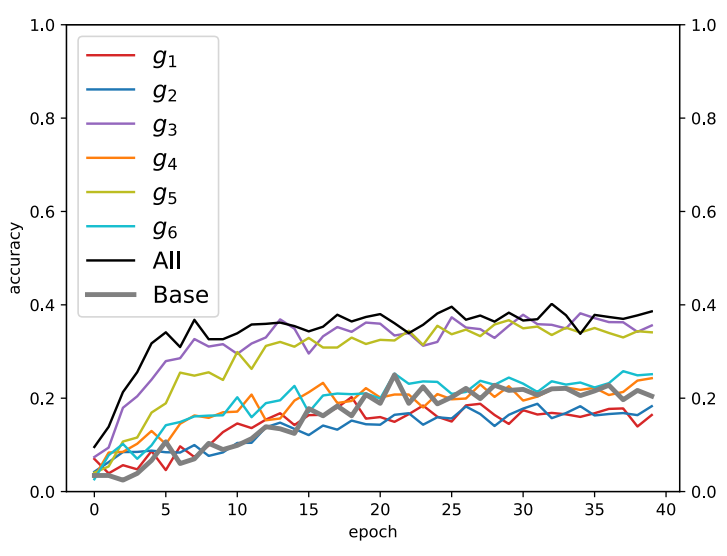

(a) Tablet $101 / b+$

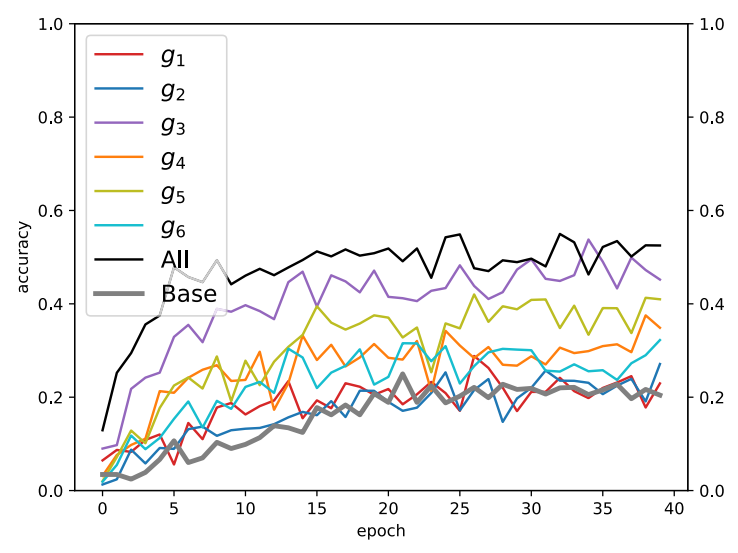

(b) Tablet Bo 49+

Fig. 12. Effect of illumination augmentation on two tablets. Colored lines represent sets of rendered data from different light directions that were added to the training set.

show that the network cannot gain useful information from these rendered images. The orange and purple lines represent groups of light directions that are present in the real photographs.

As opposed to the previous experiments, this time the light directions in $g_{6}$ contribute the most useful information to the network increasing the performance to $\tilde{9} 0 \%$. This results from most of the images in our dataset being lit from the left. Therefore, the light directions from the top do not contribute as much as the ones from the left, which results in a lower accuracy when adding data represented by the purple line. Again, the best results are achieved when incorporating all synthetic data, since the sampling of the light directions is obviously dense enough to achieve a good generalization. Regarding the total improvement, the change of the performance is not as prominent as in the previous experiments. However, the increase of 4 percentage points here corresponds a quite significant reduction of the overall classification error by $28.5 \%$. Thus, it is shown that the illumination augmentation can achieve significant improvements, even for larger, less selective cuneiform datasets. This is especially important in view of the large visual variance of the existing photographic cuneiform reproductions.

\section{CONCLUSION AND OUTLOOK}

In this article we presented and evaluated a new illumination-based augmentation technique for 2D cuneiform sign classification. Our method generates new training image data by rendering differently lit images of cuneiform signs from an annotated 3D dataset, which is then processed further and fed into a state-of-the-art image classification neural network. This way, we are able to force a classifier to learn a set of features that allows for illumination invariant classification. Our evaluation shows that our method can be used to transfer classification results between single cuneiform reproductions and small datasets with differing illumination conditions. Moreover, it can also significantly improve the performance on larger, less visually uniform cuneiform datasets. Due to the considerable reduction of manually annotated data required, this increases the accessibility of large photographic cuneiform collections to content-based retrieval methods. During the evaluation underrepresented lighting scenarios were determined manually. Future extensions of our approach could integrate methods that automatically determine underrepresented illumination directions to specifically supplement the training data. An additional target for generalization approaches is the high variance of the surface textures of cuneiform fragments. While our current approach is focused on, but not limited to, well-preserved tablets that exhibit similar surface textures, we see optimization potential in addressing the high variance of cuneiform surface textures 


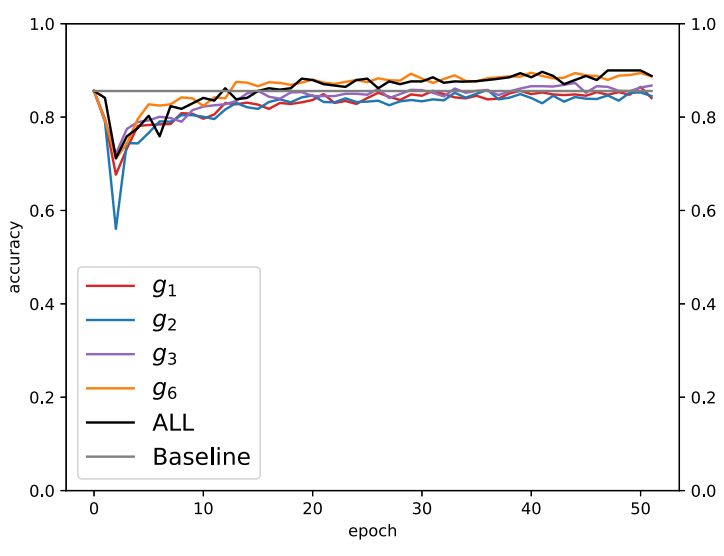

(a) All epochs

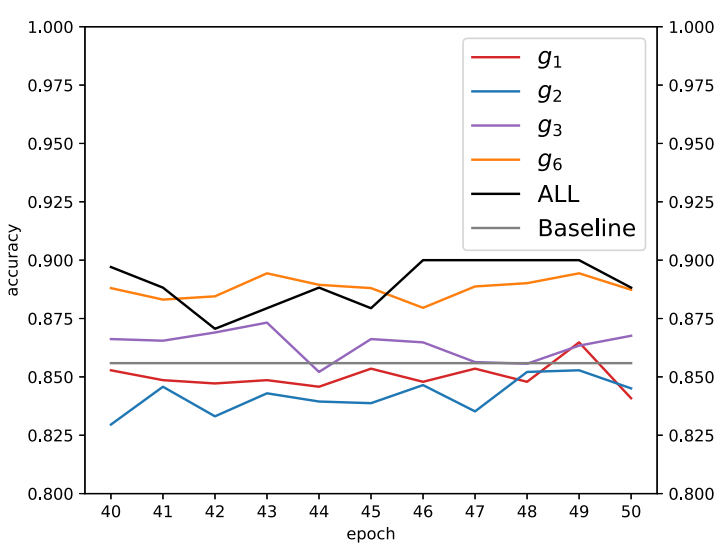

(b) Zoomed on epoch 40-50

Fig. 13. Effect of illumination augmentation using rendered data on a network that is pre-trained on photographic fragments from all tablets until convergence ( $86 \%$ accuracy). As most of our photographic dataset is illuminated from the left, the set $g_{6}$ of light directions $l_{9}, l_{10}$ has the highest impact.

(cf. Figure 1(a)) as these may complicate the training process. This could also be addressed with 3D scans as a starting point, since they provide a separable representation of geometric and texture information. During the rendering process different textures could be mapped onto the geometry to artificially create underrepresented textures, similar to the usage of different light directions as shown in this article.

In the field of cuneiform writing, Reflectence Transformation Imaging (RTI) images are common in addition to traditional photographs. While we only tested our method on traditional photographic reproductions, we think that our method can be adapted to using RTI input images instead of rendered 3D meshes as RTI images can be illuminated by a virtual light source like the rendered 3D tablets.

In addition to traditional photographs, flatbed scans are often used in the cuneiform domain. We have focused on traditional photographs and have not done any tests with flatbed scans, but the residual light present in a flatbed scan, if any is visible at all, and the resulting surface shadowing would be similar to ambient occlusion. Instead of augmenting the direction of illumination (this would have no effect since the elevation angle is $90^{\circ}$ ), ambient occlusion could be calculated for the 3D model and used with a similar surface texture of a flatbed scan to further augment data specifically for flatbed scans. However, it is unclear how well this would work.

Although our presented augmentation method is designed for cuneiform script data, it could be applied to different scenarios that exhibit different lighting conditions and in cases where 3D data are available to augment underrepresented 2D data. Possible applications could be automated manufacturing processes, where regions of interest on components, like embossed QR-codes in 3D printing, have to be localized with high confidence under varying light conditions. As 3D QR-codes, just like cuneiform wedges, become visible through shadow casting, the method presented in this article may also be helpful in the field of 3D QR code detection and help to improve detection rates. During production this could be automated with neural networks and the illumination augmentation could be used to generate new training data. 


\section{A ADDITIONAL MATERIAL}

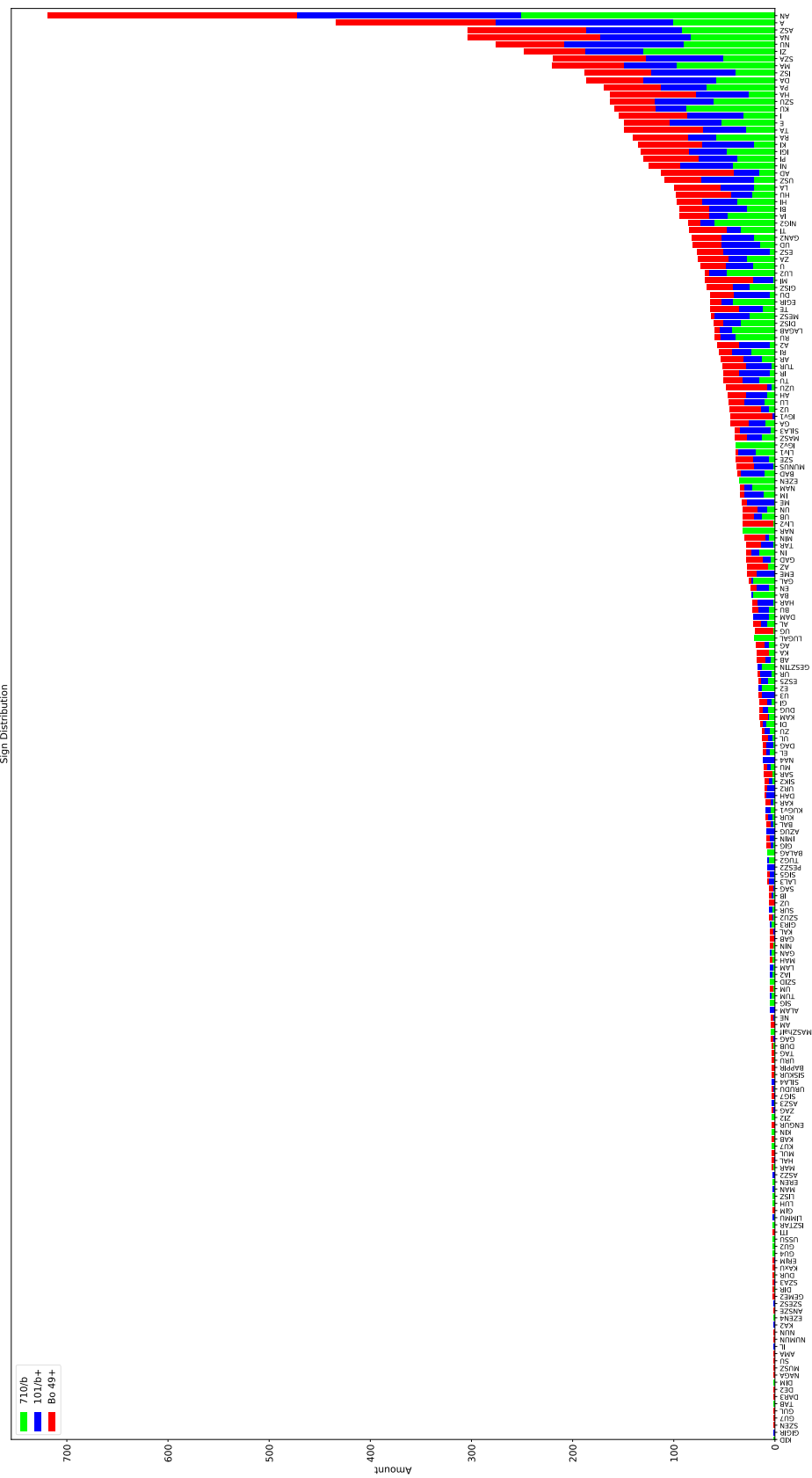

Fig. 14. Sign distribution of the tablets $710 / b, 101 / b+$, and $B o 49+$. The height shows the number of signs for each sign class for each tablet. 


\section{REFERENCES}

[1] Akm Ashiquzzaman, Abdul Kawsar Tushar, and Ashiqur Rahman. 2017. Applying data augmentation to handwritten arabic numeral recognition using deep learning neural networks. arXiv:1708.05969. Retrieved from https://arxiv.org/abs/1708.05969.

[2] Batuhan Balci, Dan Saadati, and Dan Shiferaw. 2017. Handwritten text recognition using deep learning. CS231n: Convolutional Neural Networks for Visual Recognition, Stanford University, Course Project Report, Spring (2017).

[3] Bartosz Bogacz, Michael Gertz, and Hubert Mara. 2015. Character retrieval of vectorized cuneiform script. In Proceedings of the 13th International Conference on Document Analysis and Recognition (ICDAR'15). 326-330.

[4] Bartosz Bogacz, Michael Gertz, and Hubert Mara. 2015. Cuneiform character similarity using graph representations. In Proceedings of the 20th Computer Vision Winter Workshop. 105-112.

[5] Bartosz Bogacz, Nicholas Howe, and Hubert Mara. 2016. Segmentation free spotting of cuneiform using part structured models. In Proceedings of the 15th International Conference on Frontiers in Handwriting Recognition (ICFHR'16). IEEE, 301-306.

[6] Bartosz Bogacz, Maximilian Klingmann, and Hubert Mara. 2017. Automating transliteration of cuneiform from parallel lines with sparse data. In Proceedings of the 14th IAPR International Conference on Document Analysis and Recognition (ICDAR'17), Vol. 1. IEEE, 615-620.

[7] Bartosz Bogacz and Hubert Mara. 2020. Period classification of 3D cuneiform tablets with geometric neural networks. In Proceedings of the 17th International Conference on Frontiers in Handwriting Recognition (ICFHR'20). IEEE, 246-251.

[8] Michele Cammarosano. 2014. 3D-Joins und schriftmetrologie: A quantitative approach to cuneiform palaeography. In Current Research in Cuneiform Paleography: Proceedings of a Workshop held at the 60th Rencontre Assyriologique Internationale. University of Warsaw.

[9] Michele Cammarosano. 2014. The cuneiform stylus. Mesopotamia 49 (2014), 53-90.

[10] London Department of the Middle East of the British Museum and Cuneiform Digital Library Initiative (CDLI). British Museum Collection. Retrieved June 15, 2021 from cdli.ucla.edu.

[11] Valentin Deschaintre, Miika Aittala, Fredo Durand, George Drettakis, and Adrien Bousseau. 2018. Single-image svbrdf capture with a rendering-aware deep network. ACM Trans. Graph. 37, 4 (2018), 1-15.

[12] Nikita Dvornik, Julien Mairal, and Cordelia Schmid. 2019. On the importance of visual context for data augmentation in scene understanding. IEEE Trans. Pattern Anal. Mach. Intell. 43, 6 (2019), 2014-2028.

[13] Adel I. El-Fallah and Gary E. Ford. 1997. Mean curvature evolution and surface area scaling in image filtering. IEEE Trans. Image Process. 6, 5 (1997), 750-753.

[14] Matthias Fey, Jan Eric Lenssen, Frank Weichert, and Heinrich Müller. 2017. SplineCNN: Fast geometric deep learning with continuous B-Spline kernels. arXiv:1711.08920. Retrieved from https://arxiv.org/abs/1711.08920.

[15] Denis Fisseler, Frank Weichert, Michele Cammarosano, and Gerfrid G. W. Müller. 2013. Towards an interactive and automated script feature analysis of 3D scanned cuneiform tablets. In Proceedings of the Scientific Computing and Cultural Heritage Conference (SCCH'13), Vol. 4.

[16] Denis Fisseler, Frank Weichert, Gerfrid G. W. Müller, and Michele Cammarosano. 2014. Extending philological research with methods of 3D computer graphics applied to analysis of cultural heritage. In Proceedings of the EUROGRAPHICS Workshop on Graphics and Cultural Heritage (GCH'14). 165-172.

[17] Denis Fisseler, Frank Weichert, Gerfrid G. W. Müller, and Michele Cammarosano. 2014. Extending philological research with methods of 3D computer graphics applied to analysis of cultural heritage. In Proceedings of the Eurographics Workshop on Graphics and Cultural Heritage. The Eurographics Association, 165-172.

[18] Ian Goodfellow, Yoshua Bengio, and Aaron Courville. 2016. Deep Learning. MIT Press. http://www.deeplearningbook.org.

[19] Hendrik Hameeuw and Geert Willems. 2011. New visualization techniques for cuneiform texts and sealings. Akkadica 132, 2 (2011), 163-178.

[20] Alex Hernández-García and Peter König. 2019. Further advantages of data augmentation on convolutional neural networks. arXiv:1906.11052. Retrieved from https://arxiv.org/abs/1906.11052.

[21] Gao Huang, Zhuang Liu, Laurens van der Maaten, and Kilian Q. Weinberger. 2016. Densely connected convolutional networks. arXiv:cs.CV/1608.06993. Retrieved from https://arxiv.org/abs/1608.06993.

[22] Nils M. Kriege, Matthias Fey, Denis Fisseler, Petra Mutzel, and Frank Weichert. 2018. Recognizing cuneiform signs using graph based methods. arXiv:1802.05908. Retrieved from https://arxiv.org/abs/1802.05908.

[23] Alex Krizhevsky, Ilya Sutskever, and Geoffrey E Hinton. 2012. Imagenet classification with deep convolutional neural networks. In Advances in Neural Information Processing Systems. 1097-1105.

[24] Niall O’ Mahony, Sean Campbell, Anderson Carvalho, Suman Harapanahalli, Gustavo Adolfo Velasco-Hernández, Lenka Krpalkova, Daniel Riordan, and Joseph Walsh. 2019. Deep learning vs. traditional computer vision. arXiv:1910.13796. Retrieved from https://arxiv. org/abs/1910.13796.

[25] Hubert Mara and Susanne Krömker. 2013. Vectorization of 3D-Characters by integral invariant filtering of high-resolution triangular meshes. In Proceedings of the IEEE International Conference on Document Analysis and Recognition. 62-66.

[26] Judith Massa, Bartosz Bogacz, Susanne Krömker, and Hubert Mara. 2016. Cuneiform Detection in Vectorized Raster Images. In 21st Computer Vision Winter Workshop. https://doi.org/10.11588/heidok.00020244 
[27] Gerfrid G. W. Müller. 2000. Hethitologie Portal Mainz. Retrieved May 5, 2018 from www.hethiter.net.

[28] Ismoilov Nusrat and Sung-Bong Jang. 2018. A comparison of regularization techniques in deep neural networks. Symmetry 10, 11 (2018). https://doi.org/10.3390/sym10110648

[29] Ioannis Pratikakis, Konstantinos Zagoris, Basilis Gatos, Georgios Louloudis, and Nikolaos Stamatopoulos. 2014. ICFHR 2014 competition on handwritten keyword spotting (H-KWS 2014). In Proceedings of the 14th International Conference on Frontiers in Handwriting Recognition. 814-819.

[30] Leonard Rothacker, Denis Fisseler, Gerfrid Müller, Frank Weichert, and Gernot A. Fink. 2015. Retrieving Cuneiform Structures in a Segmentation-free Word Spotting Framework. In Proceedings of the 3rd International Workshop on Historical Document Imaging and Processing. 129-136.

[31] Leonard Rothacker, Marcal Rusinol, and Gernot A. Fink. 2013. Bag-of-Features HMMs for segmentation-free word spotting in handwritten documents. In Proceedings of the International Conference on Document Analysis and Recognition. IEEE, 1305-1309.

[32] Eugen Rusakov, Kai Brandenbusch, Denis Fisseler, Turna Somel, Gernot A. Fink, Frank Weichert, and Gerfrid Müller. 2019. Generating cuneiform signs with cycle-consistent adversarial networks. In Proceedings of the International Workshop on Historical Document Imaging and Processing (HIP'19). Publikation, 19-24.

[33] Eugen Rusakov, Turna Somel, Gernot A. Fink, and Gerfrid G. W. Müller. 2020. Towards query-by-expression retrieval of cuneiform signs. In Proceedings of the 17th International Conference on Frontiers in Handwriting Recognition (ICFHR'20). IEEE, 43-48.

[34] Connor Shorten and Taghi M. Khoshgoftaar. 2019. A survey on image data augmentation for deep learning. f. Big Data 6 (2019), 1-48.

[35] Karen Simonyan and Andrew Zisserman. 2014. Very deep convolutional networks for large-scale image recognition. arXiv:1409.1556. Retrieved from https://arxiv.org/abs/1409.1556.

[36] Chen Sun, Abhinav Shrivastava, Saurabh Singh, and Abhinav Gupta. 2017. Revisiting unreasonable effectiveness of data in deep learning era. In Proceedings of the IEEE International Conference on Computer Vision. 843-852.

[37] Hugo Touvron, Andrea Vedaldi, Matthijs Douze, and Hervé Jégou. 2020. Fixing the train-test resolution discrepancy: FixEfficientNet. arXiv:2003.08237. Retrieved from https://arxiv.org/abs/2003.08237.

[38] Curtis Wigington, Seth Stewart, Brian Davis, Bill Barrett, Brian Price, and Scott Cohen. 2017. Data augmentation for recognition of handwritten words and lines using a CNN-LSTM network. In Proceedings of the 14th IAPR International Conference on Document Analysis and Recognition (ICDAR'17), Vol. 1. IEEE, 639-645.

[39] Geert Willems, Frank Verbiest, Wim Moreau, Hendrik Hameeuw, Karel van Lerberghe, and Luc van Gool. 2005. Easy and cost-effective cuneiform digitizing. In Proceedings of the 6th International Symposium on Virtual Reality, Archaeology and Cultural Heritage (VAST'05). 73-80.

[40] Saining Xie, Ross B. Girshick, Piotr Dollár, Zhuowen Tu, and Kaiming He. 2016. Aggregated residual transformations for deep neural networks. arXiv:1611.05431. Retrieved from https://arxiv.org/abs/1611.05431.

[41] Zhun Zhong, Liang Zheng, Guoliang Kang, Shaozi Li, and Yi Yang. 2017. Random erasing data augmentation. arXiv:1708.04896. Retrieved from https://arxiv.org/abs/1708.04896.

[42] George Kingsley Zipf. 1932. Selected Studies of the Principle of Relative Frequency in Language. Harvard University Press.

Received June 2021; revised October 2021; accepted November 2021 